\title{
NEW INFRARED RESULTS FOR CLASSICAL NOVAE
}

\author{
Robert D. Gehrz \\ Department of Astronomy, University of Minnesota \\ 116 Church Street S.E. \\ Minneapolis, MN 55455
}

Abstract. Recent infrared studies of classical novae are described. The nucleation and growth of dust in nova ejecta was confirmed by optical/infrared photometry of several novae during the past five years including QU Vul, V842 Cen, V827 Her, and QV Vul. These novae provided evidence that dust of every astrophysical chemical and mineral composition can condense in nova ejecta. Infrared forbidden fine structure lines were identified in the recent novae V1819 Cyg, QU Vul, OS And, and V827 Her. The implication of IRAS measurements for nova energetics is discussed.

\section{INTRODUCTION}

The most convincing cases for transient circumstellar dust formation have been constructed from infrared observations of the temporal development of classical novae (Bode 1988a and b; Bode and Evans 1989; Gehrz 1988; Starrfield 1989; contributions in this volume by A. Evans and S. Starrfield). Their infrared temporal development progresses in several identifiable stages (see Gehrz 1988 and Figure 1). The initial eruption results from a thermonuclear runaway on the surface of a white dwarf that has been accreting matter from a companion star through the inner Lagrangian point in a close binary system. Hot gas expelled in the explosion is initially seen as an expanding pseudophotosphere, or "fireball." Free-free and line emission are observed when the expanding fireba11 becomes optically thin. A dust condensation phase, characterized by rising infrared emission and a visible light transition, occurs in many novae within 50 to 200 days after the eruption. The infrared emission continues to rise as the grains grow to a maximum radius, and then falls as the mature grains are dispersed by the outflow into the ISM. The decline rate of the infrared radiation and the grain cooling rate suggest the grain radius decreases during this dispersal. About $10^{-8}$ to $10^{-6} \mathrm{M}$ of dust forms in each episode. Although most novae produce carbon dust, recent observations suggest that nova explosions can produce every other type of astrophysical grain including silicates, silicon carbide (SiC), and hydrocarbons (Gehrz 1988, 1989a and b; Hyland and MacGregor 1989). Optically thin nova shells can be strongly cooled by infrared fine structure forbidden line emission (Grasdalen and Joyce 1979; Gehrz, Grasdalen, and Hackwel1 1985; Greenhouse et al. 1988, 1989). In this paper, we review recent infrared developments in classical novae, with special emphasis on results that have been obtained during the past five years. 
a) Infrared Development Phases

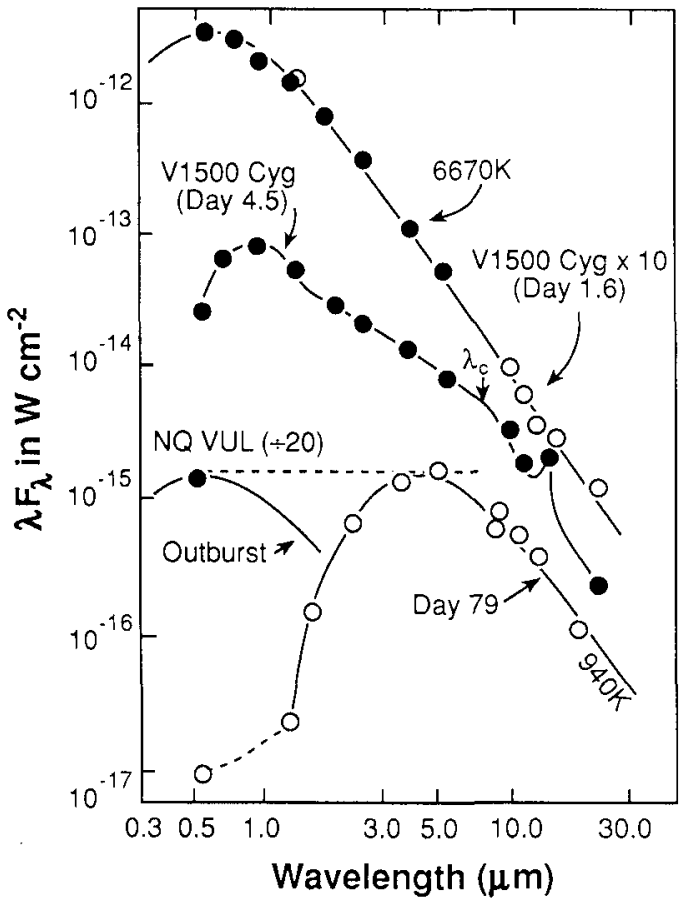

b) NQ Vul Dust Formation
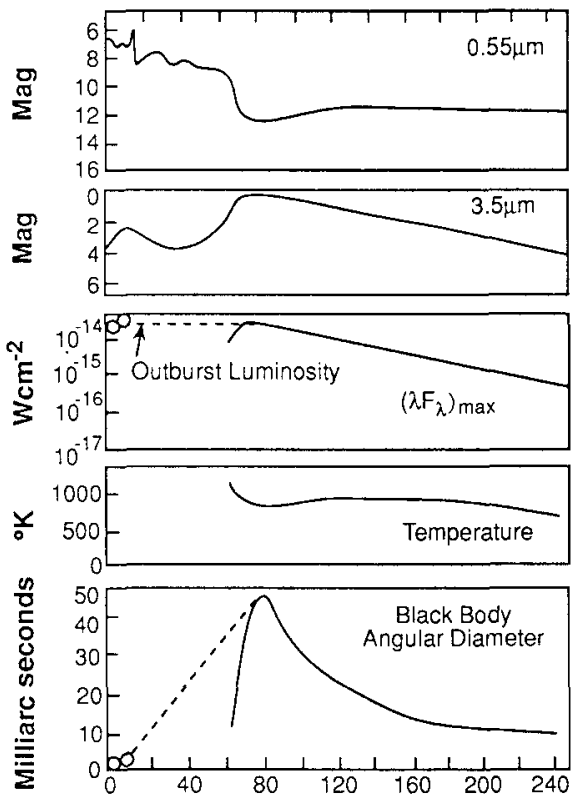

Time Since Outburst (days)

Figure 1: Infrared development phases of classical novae showing a) infrared energy distributions of the pseudophotosphere of V1500 Cyg on day 1.6 (data from Gallagher and Ney 1976 and Ennis et al. 1977), the free-free emission phase of V1500 Cyg on day 4.5 (data from Gallagher and Ney 1976), and the dust emission phase of NQ Vul on day 79 compared to its outburst pseudophotosphere (data from Ney and Hatfield 1978); and $b$ ) the classic temporal development of the dust emission phase of NQ Vul showing that the dust formed at $\approx 1000 \mathrm{~K}$ and that the dust she11 re-radiated the outburst luminosity (after Ney and Hatfield 1978). The strong decline ("transition") in visible light around day 70 coincides with dust production and a rise in the infrared light.

\section{PHYSICS OF THE EJECTA FROM RECENT INFRARED MEASUREMENTS}

Quantitative descriptions of the temporal development of the infrared energy distributions of novae in outburst, summarized briefly below, are given in detail by Ney and Hatfield (1978), Gehrz et al. (1980), Gehrz (1988) and Bode and Evans (1989). Infrared observations of the angular expansion rates of the pseudophotosphere and optically thick dust shell of a nova can be combined with the Doppler expansion velocity $V_{0}$ from visual spectroscopy to yield an accurate distance $D$ through the relationship $D=v_{0}(d \theta / d t)^{-1}$. Since the energy distribution of the nova during these phases is a blackbody (see Figure 1a) that can be characterized by a 
temperature $T_{B B}$ and a maximum emission of $\left(\lambda F_{\lambda}\right)_{\max } \mathrm{W} \mathrm{cm}^{-2}$, the angular radius in radians is $\theta=4.8 \times 10^{5}\left[\left(\lambda F_{\lambda}\right)_{\max } / T_{B B}\right]^{1 / 2}$. The luminosity $L$ of the nova then follows from $D$ and $L=4 \pi D^{2}\left[1.35\left(\lambda F_{\lambda}\right)_{\max }\right]$. The blackbody angular size method afforded by infrared measurements allows the determination of the luminosity at outburst when the energy is emitted by the pseudophotosphere, and again at later phases when the measured luminosity is that radiated by the dust she11. The largest uncertainty in this method is determining which of the visibly Doppler velocity components to assign to the principle ejecta (Gehrz 1988). The mass of the gas in the ejecta can be measured in several ways using the infrared energy distribution of the optically thin gas emission phase. When the pseudophotosphere first begins to go optically thin, the energy distribution deviates slightly from that of a blackbody, but the ejecta are so hot that the Thompson scattering opacity $k_{T}$ still dominates radiative transfer in the shell. In this case, the shell mass is given by $M-\pi R^{2} K_{T}{ }^{-1}$ where the shell radius $R=t V_{0}$ and $t$ is the time since outburst. Later, when the ejecta cool sufficiently that thermal Bremstrahlung dominates the shell opacity, the mass of a shell of thickness $\iota(p c)$ and electron density $\mathrm{n}_{e}\left(\# \mathrm{~cm}^{-3}\right)$ can be determined through the relationship $n_{e}{ }^{2} \lambda_{c}{ }^{2} \iota=10^{18}$ and $M \approx 4 \pi R^{2} \iota n_{e}$ where $\lambda_{c}(\mathrm{~cm})$ is the "cutoff" wavelength at which the free-free optical depth becomes unity (see Figure 1a).

The temporal development of the optical/infrared energy distributions (see Figure $1 \mathrm{~b}$ ) of dusty novae is consistent with a scenario in which dust formation occurs at a temperature $\mathrm{T}_{0} \approx 1000 \mathrm{~K}$ when the outflowing ejecta reach the condensation radius $\mathrm{R}_{0}=\left[\mathrm{L}_{0} / 16 \pi \sigma \mathrm{T}_{0}{ }^{4}\right]^{1 / 2}$. If the outflow velocity $\mathrm{V}_{0}$ is constant, then the dust formation should occur on a timescale of $t_{d}=R_{0} / V_{0}=137\left(\mathrm{~L} / \mathrm{L}_{\odot}\right)^{1 / 2} \mathrm{~V}_{0}^{-1}$ days and the shell density at the condensation point will be $\rho_{c} \approx 1.2 \times 10^{-4}\left(\mathrm{M} / \mathrm{M}_{\odot}\right)\left(\mathrm{L} / \mathrm{L}_{\odot}\right)^{-3 / 2} \mathrm{gm} \mathrm{cm}^{-3}$. Novae that produce optically thick dust shells apparently have a "critical" shell density at the condensation point of $\rho_{c}=2 \times 10^{-16}$ to $10^{-15} \mathrm{gm} \mathrm{cm}^{-3}$ (see Table 1). The onset of visible transitions (Figure 1b) in dusty novae for which the outburst time is well determined is entirely consistent with the timescale $t_{d}$. It is evident from Table 1 that the time for the grains to reach maximum growth is 2 to 3 times as long as $t_{d}$ for novae that grow optically thick she1ls, and less than 2 times as long in cases where the shells do not become optically thick. This no doubt reflects the fact that shell densities stay high longer in novae that produce optically thick shells, so that the grain growth can proceed for longer times. Obscuration of the central engine by the dust grains causes the visual transition (Figure $1 \mathrm{~b}$ ), and the corresponding increase in infrared intensity comes from thermal reradiation by the grains of the energy they absorbed. The time of $\leq 37$ days in which V1370 Aq1 produced an optically thick dust shell was remarkably short reckoned from the discovery date, but there is reason to suspect that the ejecta of this nova had an exceptionally high expansion velocity so that $t_{d}$ would be expected to be small (Gehrz et al. 1984). An elementary application of kinetic theory to the data shows that the grains in dusty novae grow to sizes of 0.1 to 1 micron within 10 to 100 days. The grains cease growing eventually because the condensation process is cut 
off by the decreasing density of the expanding shell. Following growth to their maximum radius, grains in the outflow apparently are processed to smaller sizes by sputtering or evaporation. Recovery of the visual light from the "transition" phase (see Figure 1b) occurs as the outflowing shell, the total absorption cross section of its grain mass now constant or decreasing, becomes optically thin to radiation from the central engine. The most vexing problem in grain growth models is to provide seed nuclei given the extremely harsh radiation environment in the ejecta. Various refractory seed nuclei and molecular species that can accrete into seed nuclei have been proposed (see Gehrz 1988; Rawlings 1988). A novel suggestion by Matese, Whitmire, and Reynolds (1989) is that the outburst can release a significant amount of preexisting dust from comet nuclei in a cometesimal disk, and that this material provides seed nuclei that then accrete material in the ejecta. Dust released from planetesimals in sufficient quantities could even cause a sudden obscuration of the visible light from the remnant; it is well known that the opacity of a given circumstellar mass of solid planetesimals increases as $a^{-1}$ where a is the radius of the average planetesimal (see Gehrz 1989b).

\section{DUST IN RECENT NOVAE}

Table 1 summarizes dust formation in novae as recorded by infrared measurements since 1970. Thirteen of 21 recent bright classical novae are known with certainty to have produced measurable amounts of dust. The remaining eight either produced traces of dust that were undetected, or were not observed with sufficient diligence (in temporal, spectral, or photometric coverage) to provide conclusive evidence of dust production. For example, V1500 Cyg is known to have emitted excess $10 \mu \mathrm{m}$ radiation after 100 days (Ennis et al. 1977), but the low spectral resolution of the measurements does not allow any definitive conclusions about the origin of the excess. Seven of the thirteen dust forming novae produced optically thick dust shells that reradiated at least half of the outburst luminosity. We conclude that at least one-third of all novae produce optically thick dust shells.

The dust formation hypothesis has been confirmed for several recent novae. V827 Her (Gehrz and Jones 1987) and LMC Nova 1988\#1 (Whitelock 1988) formed optically thin dust she11s, while V842 Cen (Figure 2) and QV Vul (Figure 3) formed optically thick dust shells. V842 Cen was remarkable for the very fast, deep minimum it showed during the visible transition phase (see Figure 2a). Since the integrated absorption optical depth of the dust shell was still nearly unity after 500 days (Figure $2 \mathrm{~b}$ ), the deep minimum may have been caused by a relatively small highly opaque structure that passed across the line of sight for a short time during the early development of the dust she1l. Such structures could be related to the polar plumes and equatorial ring believed to form in the eruption (see Gallagher and Starrfield 1978). 
TABLE 1: SUMMARY OF DUST FORMATION IN 21 RECENT CLASSICAL NOVAE

\begin{tabular}{|c|c|c|c|c|c|c|}
\hline Name & Year & $\begin{array}{c}v_{0} \\
\left(k m s^{-1}\right)\end{array}$ & $\begin{array}{l}\text { Dust Optica } \\
\text { Depth } \\
\mathrm{T}=\frac{\left(\mathrm{L}_{\mathrm{IR}}\right)_{\max }}{\left(\mathrm{L}_{\mathrm{o}}\right)_{\max }}\end{array}$ & $\begin{array}{l}\frac{\left(t_{d}\right)}{t_{I R \max }} \\
\text { days }\end{array}$ & $\begin{array}{c}\text { Shell } \\
\text { Density at } \\
\mathrm{R}_{\mathrm{c}}\left(\mathrm{gm} \mathrm{cm}^{-2}\right)\end{array}$ & $\begin{array}{l}\text { Dust Types } \\
\text { Formed }\end{array}$ \\
\hline FH Ser & 1970 & 560 & 0.50 & 60 & - & $\mathrm{C}$ \\
\hline V1229 Aql & 1970 & 575 & 0.55 & - & - & $\mathrm{C}$ \\
\hline $\mathrm{V} 1301 \mathrm{Aq} 1$ & 1975 & - & - & - & - & $\mathrm{C}$ \\
\hline V1500 Cyg ${ }^{2}$ & 1975 & 1180 & $?$ & $100 / 100$ & $3 \times 10^{-17}$ & - \\
\hline NQ Vu1 & 1976 & 750 & 1.00 & $32 / 80$ & $2.3 \times 10^{-15}$ & $\mathrm{C}$ \\
\hline $\mathrm{V} 4021 \mathrm{Sgr}$ & 1977 & - & $\leq 0.60$ & - & - & $\mathrm{C}$ \\
\hline LW Ser & 1978 & 1250 & 0.70 & $23 / 75$ & $3.0 \times 10^{-16}$ & $\mathrm{C}$ \\
\hline v1668 Cyg & 1978 & 1300 & 0.08 & $33 / 57$ & $7.6 \times 10^{-17}$ & $\mathrm{C}$ \\
\hline $\mathrm{V} 1370 \mathrm{Aq} 1^{4}$ & 1982 & 2800 & 0.50 & $\leq 16 / \leq 37$ & - & $\mathrm{C}, \mathrm{SiC}, \mathrm{SiO}_{2}$ \\
\hline GQ Mus & 1983 & 600 & no dust & - & $\leq 10^{-17}$ & no dust \\
\hline PW Vul & $1984 \# 1$ & 285 & $3 \times 10^{-3}$ & $152 / \leq 280$ & $2.0 \times 10^{-17}$ & C \\
\hline QV $V u 1^{2}$ & $1984 \# 2$ & $1-5000$ & $3 \times 10^{-3}$ & $40-200 / 240$ & - & $\mathrm{SiO}_{2}$ \\
\hline os And ${ }^{2}$ & 1986 & 900 & no dust & - & $1.1 \times 10^{-17}$ & no dust \\
\hline V1819 $\mathrm{Cyg}^{2}$ & 1986 & 1000 & no dust & - & - & no dust \\
\hline V842 Cen & 1986 & 1200 & 1.00 & $36 / 87$ & - & $\mathrm{C}, \mathrm{SiO}_{2}, \mathrm{HC}$ \\
\hline V827 Her $^{2}$ & 1987 & 1000 & 0.10 & $43 / 83$ & - & C \\
\hline V4135 Sgr & 1987 & 500 & - & - & - & - \\
\hline QV Vul & 1987 & 700 & 1.00 & $56 / 115$ & $2.0 \times 10^{-16}$ & $\mathrm{C}, \mathrm{SiO}_{2}, \mathrm{HC}$ \\
\hline IMC $1988 \# 1$ & $1988 \# 1$ & 800 & 0.06 & $59 / 57$ & - & $\mathrm{C} ?$ \\
\hline LMC $1988 \# 2$ & $1988 \# 2$ & 1500 & - & - & - & - \\
\hline v2214 Oph & 1988 & 500 & - & - & - & - \\
\hline
\end{tabular}

${ }^{1}$ Data from Gehrz 1988, references in Figures 2, 3, and IAU Circulars.

${ }^{2}$ Infrared coronal emission lines recorded.

${ }^{3} \mathrm{HC}=$ Hydrocarbons

${ }^{4}$ The $7-14 \mu \mathrm{m}$ emission may have been caused by a combination of $\mathrm{SiC}$ and $\mathrm{SiO}_{2}$.

Although most novae form carbon dust, there is now evidence that astrophysical dust of every known chemical and mineral composition can condense in nova ejecta (Table 1). QU Vul (Gehrz et a1. 1986), V842 Cen (Figure 2d), and QV Vul (Figure 2b) formed silicate grains, V1301 Aql may have formed SiC or a combination of SiC and silicates (see Bode et al. 1984; Gehrz et al. 1984), and V842 Cen (Figure 2c), and QV Vul (Greenhouse et al. 1989) formed hydrocarbon grains. The hard radiation from the nova remnant may provide the radiation processing that is believed to be important in creating hydrocarbons from amorphous carbon (Allamandola 1984; Tielens and Allamandola 1987). While the 3.28 and $3.4 \mu \mathrm{m}$ features in QV Vul and V842 Cen are most probably caused by $\mathrm{C}-\mathrm{H}$ stretch emission from hydrocarbons, the $11.3 \mu \mathrm{m}$ feature 
in V842 Cen could be caused either by hydrocarbons or an annealed olivine silicate grain component (Aitken et a1. 1988).

The formation of more than one type of grain has now been recorded for at least three novae. Both V842 Cen (Figure 2) and QV Vul (Figure 3) apparently formed
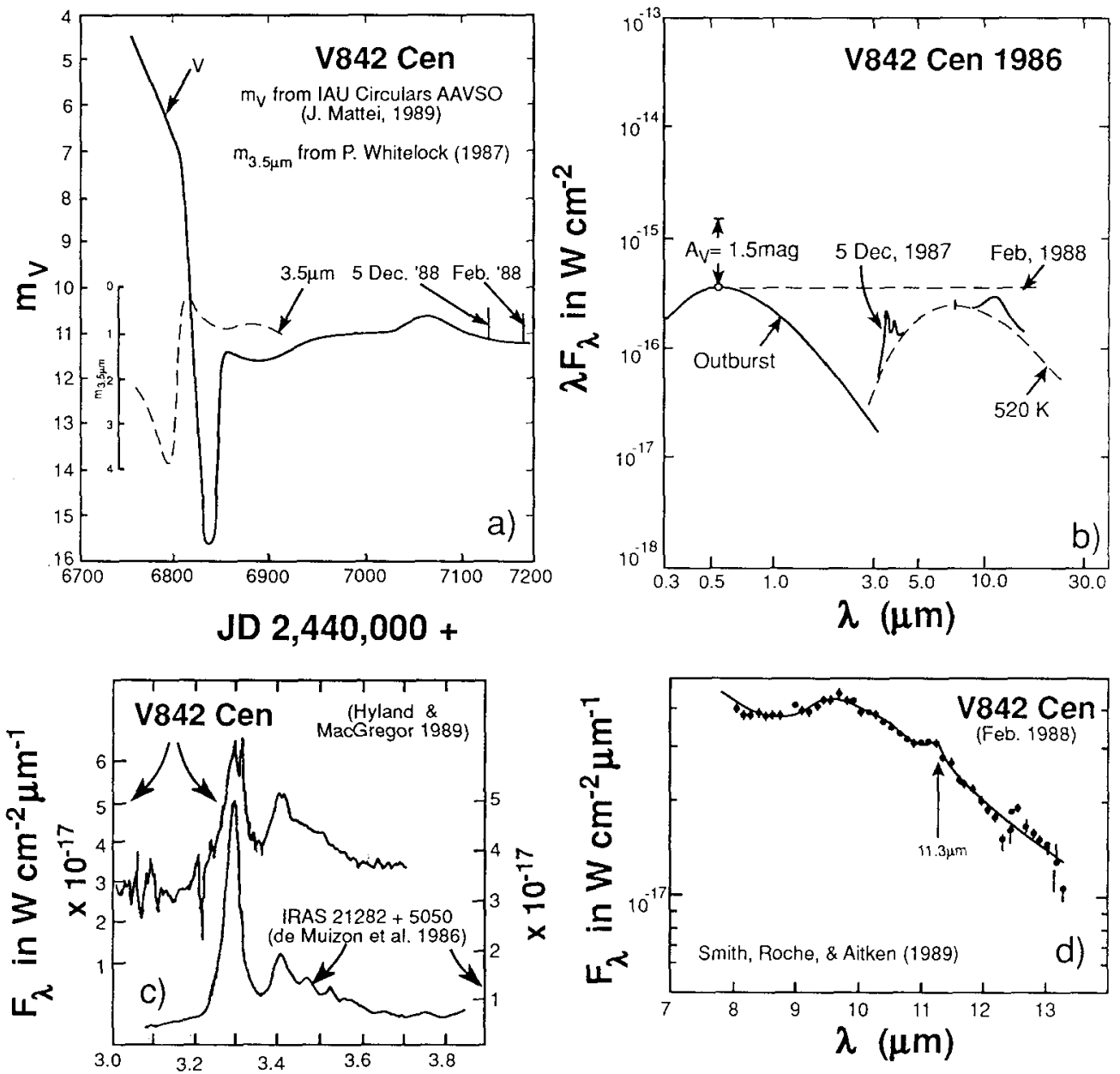

Figure 2: Nova Centaurus 1986 displayed one of the most bizarre dust formation scenarios ever observed in a classical nova. a) The visible transition was remarkably sharp with a deep short-lived minimum, yet b) the dust shell luminosity was still within a factor of 1.2-3 (depending upon the interstellar reddening) of the outburst luminosity as late as 500 days after the outburst. In addition, at least three different types of dust appear to have formed in the ejecta. c) The presence of Hydrocarbons is suggested by emission features at $3.28,3.4$, while d) carbon and silicates are required to fit the broad $7-13 \mu \mathrm{m}$ emission feature (Smith, Roche, and Aitken 1989). The $11.3 \mu \mathrm{m}$ feature could be due either to hydrocarbons or annealed olivine (see text). Possible reddening is indicated by $A_{v}$. 

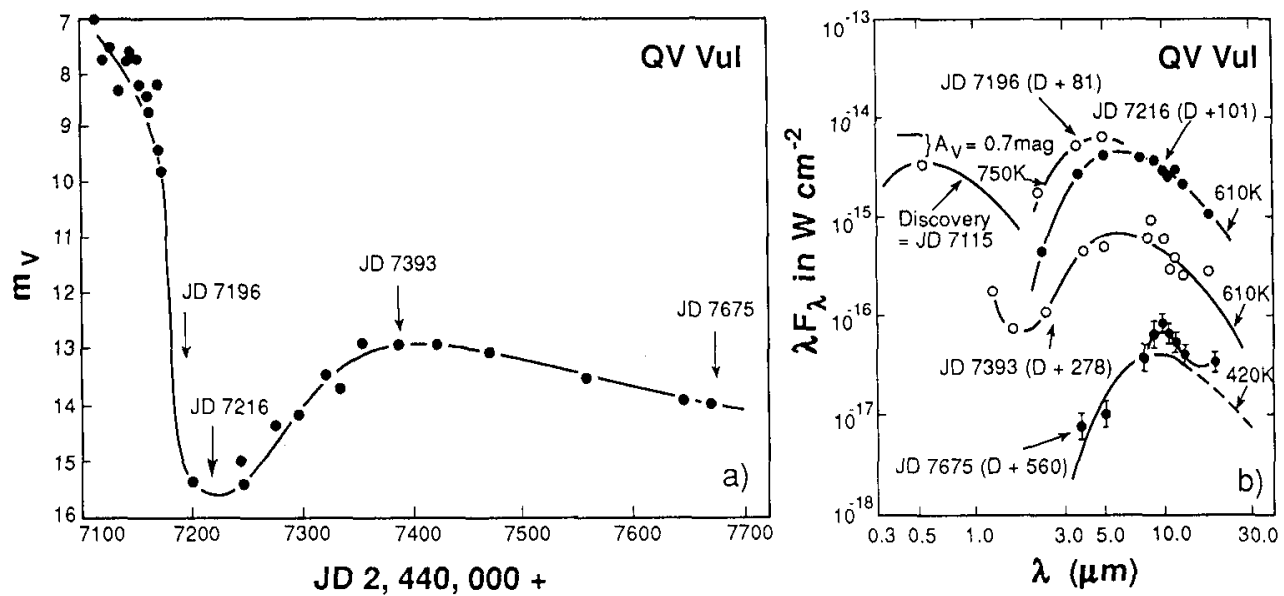

Figure 3: Nova Vulpeculae 1987 formed an optically thick dust shell that caused a) a deep visible transition, and b) still re-radiated the outburst luminosity after 100 days. Apparently, both carbon and silicate grains formed in the ejecta as evidenced by a smooth continuum (day 101) and emission features at 10 and $20 \mu$ m (day 560 ). Visible data are from IAU Circulars and the AAVSO (Mattei 1989); infrared data are from R.D. Gehrz et al. unpublished data). Possible reddening is indicated by $A$.

carbon, silicates, and hydrocarbons. It is difficult to understand how both silicates and carbon could form co-spatially in the ejecta since the silicate condensation requires an environment where $O>C$ and carbon condensation requires a gas composition with $\mathrm{C}>0$. The implication is that there must be significant abundance gradients within the ejecta. Perhaps the polar plumes and equatorial ring that are produced in the explosion can have different chemical compositions.

\section{PHYSICS OF THE INFRARED CORONAL PHASE}

Recent infrared spectroscopic observations (see Grasdalen and Joyce 1976 , Benjamin and Dinerstein 1989, Geballe 1989, Greenhouse et al. 1987, 1989) of infrared forbidden fine structure lines in V1500 Cyg, V1819 Cyg, QV Vul, OS And, and V827 Her, have confirmed a theoretical prediction by Ferland and Shields (1978) that such emission might be an important source of cooling in nova shells. The strongest of these lines was $12.8 \mu \mathrm{m}$ [Ne II] emission from QV Vul (Gehrz et al. 1985).

Infrared spectroscopic information can be important for the estimation of abundances of atomic and molecular species in nova ejecta. Greenhouse et al. (1988, 1989) have established the importance of the infrared coronal emission phase in classical novae for estimating physical conditions in the ejecta, and for determining chemical abundances related to the nucleosynthesis of the nova progenitor. These observations may also provide information about the nucleosynthesis occurring in the thermonuclear runaway during the nova eruption. While the infrared transitions 
themselves are collisionally excited, the ionization states responsible for the transitions must be largely radiatively excited (see Krautter and Williams 1987; Greenhouse et al. 1989). In some cases, it seems possible that a collisional excitation component may be required to explain the ionization structure (Greenhouse et al. 1989). Excitation temperatures inferred for the photoionization of the coronal states are as high as 500,000 - 1,000,000K. Infrared speckle interferometric measurements show that the coronal emission occurs in the principle ejecta rather than close to the central engine (Greenhouse et al. 1989). High resolution near infrared spectroscopy gives velocity widths for coronal lines that are consistent with their production in the principle ejecta (Greenhouse et al. 1989).

Analyses of the abundances of the coronal species show that the ejecta of these novae are rich in $\mathrm{O}, \mathrm{Ne}$, and $\mathrm{Mg}$. The infrared coronal emission lines in QU Vul (Gehrz et al. 1985 and Greenhouse et al. 1988) lead to the conclusion that its ejecta may be overabundant in neon and silicon (Gehrz et al. 1984; Gehrz, Grasdalen and Hackwell 1985; Williams et a1. 1985; Gehrz et al. 1987). Starrfield, Sparks, and Truran (1985), and Truran and Livio (1986) have argued that these novae result from accretion of matter onto ONeMg white dwarfs that are the evolutionary end product of intermediate mass $\left(10 \mathrm{M}_{\odot}\right)$ stars. The explosive nucleosynthesis in such systems can produce significant quantities of the radioactive isotopes ${ }^{22} \mathrm{Na}$ and ${ }^{26} \mathrm{~A} 1$ which beta decay into ${ }^{22} \mathrm{Ne}(2.7$ years $)$ and ${ }^{26} \mathrm{Mg}\left(7.3 \times 10^{5}\right.$ years). Recently, Higdon and Fowler $(1987,1989)$ have discussed the ${ }^{22} \mathrm{Na}$ and ${ }^{26} \mathrm{Al}$ yields that might be expected from Galactic Classical Novae. These materials could be trapped in grains during the dust formation phase of novae. Inclusions with significant enrichments of ${ }^{22} \mathrm{Ne}$ and ${ }^{26} \mathrm{Mg}$ have been discovered in solar system meteorites (see Gehrz 1988 and Truran 1985). One interpretation is that the grains comprising these meteoritic inclusions were condensed in the vicinity of sources of explosive nucleosynthesis such as novae or supernovae (see Clayton 1982, Truran 1985, and Gehrz 1988). Theoretical studies suggest that these abundances may reveal important information about nucleosynthesis during both the outburst and/or the evolution of the progenitor. Ballester, Antoniewicz, and Smoluchowski (1989), Kroto (1988), and Kroto and McKay (1988) have argued that carbon cages (fullerines) that may be produced in circumstellar outflows can trap ${ }^{22} \mathrm{Na}$. Kroto (1988) has also pointed out that hydrogenated fullerines could explain the hydrocarbon $\mathrm{C}-\mathrm{H}$ stretch emission seen in astrophysical sources.

\section{NOVA ENERGETICS FROM RECENT INFRARED OBSERVATIONS}

Infrared observations of the energy distributions of the optically thick dust shells of V842 Cen (Figure 2) and QV Vul (Figure 3) confirm that the central engine maintains a constant luminosity close to the Eddington limit for at least several hundred days following the eruption. The constant luminosity phase persisted for at least 100 days in QV Vul and for over 500 days in V842 Cen. IRAS observations of 
novae (Dinerstein 1986; Callus et al. 1987, and Harrison and Gehrz 1988) have led to interesting speculation on the late stages of the evolution of the nova eruption and on the nature of the accretion phase. At least several old novae and nova precursors have surprisingly large far infrared emission which could be due to either dust emission or fine structure line emission. The central engine required to power this emission must in some cases be 10 to 100 times more luminous than current theories of the accretion phase would predict. Several possibilities are that 1 ) the constant luminosity phase lasts for many years, and that 2) the boundary layer radiation comes out at EUV and X-ray wavelengths (see Ferland et al. 1982).

\section{ACKNOWLEDGEMENTS}

The author is supported by NASA, the United States Air Force, the National Science Foundation, and the University of Minnesota Graduate School.

\section{REFERENCES}

Aitken, D.K., Roche, P.F., Smith, C.H., James, S.D., and Hough, J.H. 1988, M.N.R.A.S., 230, 629-638,

Allamandola, L.J. 1984, in Galactic and Extragalactic Infrared Spectroscopy, eds. M. F. Kessler and J.P. Phillips, Dordrecht: Reide1, pp. 5-35.

Benjamin, B., and Dinerstein, H.L. 1989, private communication of work in progress.

Bode, M.F. 1988a, in Dust in the Universe, eds. M. E. Bailey and D.A. Williams, Cambridge University Press: London, pp. 73-102.

Bode, M.F. 1988b, in Infrared Spectroscopy in Astronomy, eds. M. Kessler and C. Glasse, ESA-SP for ESLAB 22, in press.

Bode, M.F., and Evans, A. 1989, in Classical Novae, eds. M.F. Bode and A. Evans, John Wiley and Sons Ltd.: London, pp. 163-186.

Bode, M.F., Evans, A., Whittet, D.C.B., Aitken, D.K., Roche, P.F., and Whitmore, B. 1984, M.N.R.A.S., 207, 897-907.

Callus, C.M., Evans, A., Albinson, J.S., Mitche11, R.M., Bode, M.F., Jameson, R.F., King, A.R., and Sherrington, M. 1987, M.N.R.A.S., 229, 539-548.

Clayton, D.D. 1982, Q.J.R. Astron. Soc. (UK), 23, 174-212.

Clayton, D.D. 1984, Ap.J., 280, 144.

de Muizon, M., Geballe, T.R., d'Hendecourt, L.B., and Baas, F. 1986, Ap.J. (Letters), 306, L105-L108.

Dinerstein, H.L. 1986, A.J., 92, 1381-1386.

Ennis, D., Becklin, E.E., Beckwith, S., Elias, J., Gatley, I., et al. 1977, Ap.J., $214,478-87$.

Ferland, G.J., and Shields, G.A. 1978, Ap.J. (Letters), 224, L15-L18.

Ferland, G.J., Langer, S.H., MacDonald, J., Pepper, G.H., Shaviv, G., and Truran, J.W. 1982, Ap. J. (Letters), 262, L53-L58.

Gallagher, J.S., and Ney, E.P. 1976, Ap.J. (Letters), 204, L35-L39.

Gallagher, J.S., and Starrfield, S.G. 1978, Ann. Rev. Astron. Astrophys., 16, 171.

Geballe, T. 1989, private communication.

Gehrz, R.D. 1988, Ann. Rev. Astron. Astrophys., 26, 377-412.

Gehrz, R.D. 1989a, in Interstellar Dust: Proceedings of IAU Symposium No. 135, eds. L. Allamandola and A.G.G.M. Tielens (Reidel ordrecht), in press.

Gehrz, R.D. 1989b, in Proceedings of the NAS/ASUSSR Workshop on Planetary Sciences, National Academy Press: Washington DC, in press.

Gehrz, R.D., Grasdalen, G.L., Greenhouse, M.A., Hackwe11, J.A., Hayward, T., and Bentley, A.F. 1986, Ap.J. (Letters), 308, L63.

Gehrz, R.D., Grasdalen, G.L., and Hackwe11, J.A. 1985, Ap.J. (Letters), 298, L47; erratum 1986, Ap.J. (Letters), 306, L49. 
Gehrz, R.D., Grasdalen, G.L., Hackwel1, J.A., and Ney, E.P. 1980, Ap.J., 237, $855-$ 865 .

Gehrz, R.D., and Jones, T.J. 1987, IAU Circular No. 4371.

Gehrz, R.D., Ney, E.P., Grasdalen, G.L., Hackwe11, J.A., and Thronson, H.A. 1984, Ap. J., 281, 303-312.

Grasdalen, G.L., and Joyce, R.R. 1976, Nature, 259, 187-189.

Greenhouse, M.A., Grasdalen, G.L., Hayward, T.L., Gehrz, R.D., and Jones, T.J. 1988, A. J., 95, 172-177

Greenhouse, M.A., Grasdalen, G.L., Woodward, C.E., Benson, J., Gehrz, R.D., Rosenthal, E., and Skrutskie, M.F. 1989, Ap.J., in press.

Harrison, T.E., and Gehrz, R.D. 1988, A.J., 96, 1001-1010.

Higdon, J.C., and Fowler, W.A. 1987, Ap.J., 317, 710-716.

Higdon, J.C., and Fowler, W.A. 1989, Ap.J., 339, in press.

Hyland, A.R., and MacGregor, P.J. 1989, in Interstellar Dust: Contributed Papers, eds. L. Allamandola and A.G.G.M. Tielens (Reidel Dordrecht), NASA Conference Publication, in press.

Krautter, J., and Williams, R.E. 1989, Ap.J., in press.

Kroto, H.W. 1988, Science, 242, 1139-1145.

Kroto, H.W., and McKay, K. 1988, Nature, 331, 328-331.

Matese, J.J., Whitmire, D.P. and Reynolds, R.T. 1989, Icarus, in press.

Mattei, J.A. 1989, AAVSO observations, private communication.

Ming, T., Anders, E., Hoppe, P., and Zinner, E. 1989, Nature, submitted.

Ney, E.P., and Hatfield, B.F. 1978, Ap.J. (Letters), 219, L111.

Rawlings, J.M.C. 1988, M.N.R.A.S., 232, 507-524.

Smith, C., Roche, P.F., and Aitken, D. 1989, private communication.

Starrfield, S.G. 1989, in Multiwavelength Studies in Astrophysics, ed. F. Cordova, Cambridege: Cambridge Univ. Press, in press.

Starrfield, S.G., Sparks, W.M., and Truran, J.W. 1985, Ap.J., 291, 136-146.

Starrfield, S.G., Sparks, W.M., and Truran, J.W. 1986, Ap.J. (Letters), 303, L5-L9.

Tielens, A.G.G.M., and Allamandola, L.J. 1987, in Interstellar Processes, eds.

D.J. Hollenbach and H.A. Thronson, Jr., Dordrecht: Reidel, pp. 397-469.

Truran, J.W. 1985, in "Nucleosynthesis: Challenges and New Developments", pp. 292.

Truran, J.W., and Livio, M. 1986, Ap.J., 308, 721-727.

Whitelock, P. 1987, "Nova Gen 1986", MNASSA, 46, pp. 72-74.

Whitelock, P. 1988, IAU Circ. No. 4601

Williams, R.E., Ney, E.P., Sparks, W.M., Starrfield, S.G., Wyckoff, S., et al. 1985, M.N.R.A.S., 212, 753-766. 